\title{
Molecular association of functioning stroma with carcinoma cells in the ovary: A preliminary study
}

\author{
MIKA NARIKIYO $^{1}$, MITSUTAKE YANO $^{2,3}$, KOUICHI KAMADA $^{2}$, \\ TOMOMI KATOH ${ }^{2}$, KOZUE ITO ${ }^{2}$, MASAYO SHUTO ${ }^{1}$, \\ HIDEKAZU KAYANO $^{1}$ and MASANORI YASUDA ${ }^{2}$
}

\author{
${ }^{1}$ School of Medical Technology, Faculty of Health and Medical Care, Saitama Medical University; \\ ${ }^{2}$ Department of Pathology, Saitama Medical University International Medical Center, Hidaka, Saitama 350-1298; \\ ${ }^{3}$ Department of Obstetrics and Gynecology, Oita University Faculty of Medicine, Oita 879-5593, Japan
}

Received August 27,2018; Accepted January 23, 2019

DOI: $10.3892 / \mathrm{ol} .2019 .9992$

\begin{abstract}
The cancer stroma serves an important role in tumour behaviours, including invasion, metastasis, and response to chemotherapy. The stroma of ovarian carcinoma is sometimes specialized, with luteinisation and/or hyperthecosis, and is designated as the 'functioning stroma' because it exerts endocrine function and produces sex steroid hormones. In the present study, 14 ovarian cancers with functioning stroma, comprising 7 endometrioid carcinomas and 7 clear cell carcinomas, were analysed to evaluate the molecular association of the functioning stroma with carcinoma cells. The median age of the patients was 67 years (range, 52-85 years); 13 patients were postmenopausal, and one was in perimenopause. Serum oestrogen values ranged from 10 to $129 \mathrm{ng} / \mathrm{ml}$, with a median of $51 \mathrm{ng} / \mathrm{ml}$. Sequence abnormalities in AT-rich interaction domain 1A (ARIDIA), phosphatidylinositol-4,5-bisphosphate 3-kinase catalytic subunit $\alpha$ (PIK3CA), Kirsten rat sarcoma viral proto-oncogene (KRAS) and phosphatase and tensin homolog (PTEN) were examined in whole tumours. For cancers positive for sequence abnormalities, their localization in carcinoma cells and/or stromal cells was examined. A total of 8 mutations - ARIDIA (L2155L), PIK3CA (H1047R), KRAS (Q12V, E31K, Q61L), and PTEN (C105fs*8) - were identified in the whole tumours of
\end{abstract}

Correspondence to: Professor Masanori Yasuda, Department of Pathology, Saitama Medical University International Medical Center, 1397-1 Yamane, Hidaka, Saitama 350-1298, Japan

E-mail: m_yasuda@saitama-med.ac.jp

Abbreviations: EOC, epithelial ovarian cancer; EMC, endometrioid carcinoma; CCC, clear cell carcinoma; FIGO, The International Federation of Obstetrics and Gynecology; FSH, follicle-stimulating hormone

Key words: ovarian cancer, functioning stroma, gene mutations, Kirsten rat sarcoma viral proto-oncogene, AT-rich interaction domain 1A, phosphatidylinositol-4,5-bisphosphate 3-kinase catalytic subunit $\alpha$, phosphatase and tensin homolog
5 patients. Seven of these eight mutations were detected only in carcinoma cells. However, one case of endometrioid carcinoma had a $K R A S(E 31 K)$ mutation in both carcinoma and stromal cells. In conclusion, although functioning stromal cells of ovarian cancer are usually thought to be non-neoplastic, some may share an origin with carcinoma cells.

\section{Introduction}

The cancer stroma, which is generally thought to be derived from non-neoplastic cells represented by cancer-associated fibroblasts, plays an important role in various tumour behaviours, such as invasion, metastasis, and chemotherapeutic response (1-3). Carcinoma-associated fibroblasts promote cancer progression (4), angiogenesis (5), and metastasis (6). In several cancers, interactions between cancer cells and the associated stroma lead to drug resistance (7). Recent studies have suggested that some carcinoma cells and stromal cells may share an origin, based on gene landscapes shared by both cell types in certain cancers, including breast (8), colon (9), bladder $(10)$, and ovarian cancers $(11,12)$. However, the origin and role of cancer stroma have been controversial yet.

Epithelial ovarian cancer (EOC) is the leading cause of death arising from gynecological malignancies (13). EOCs, including serous carcinoma, endometrioid carcinoma (EMC), clear cell carcinoma (CCC), and mucinous carcinoma, have specific clinical and genetic features. EMC and CCC are histogenetically associated with endometriosis and are often characterized by $A R I D I A$ (30 and 40-60\%, respectively), PIK3CA (40 and 51\%, respectively), KRAS (33 and 20\%, respectively), and PTEN (17 and 13\%, respectively) (14-17). But the frequency of TP53 mutation in EMC and CCC are considerably lower (7 and 13\%, respectively), compared to the four genes (14-17). The stroma of EOCs sometimes consists of a specialized ovarian stroma with luteinisation and/or hyperthecosis with endocrine function, called a 'functioning stroma' (18). The relationship between functioning stroma and the response to chemotherapy or prognosis remains to be clarified. In addition, the histogenetic mechanism of functioning stroma is poorly defined. Functioning stroma is observed not only in mucinous carcinoma but also in 
EMC and CCC (19). However, serous carcinoma characterized by TP53 mutation rarely has a functioning stroma (19). Therefore, this study aimed to evaluate the localization of gene abnormalities commonly detected in EMC and CCC in carcinoma cells and functioning stromal cells separately. We believe that some of functioning stroma may share an origin with carcinoma cells.

\section{Materials and methods}

Patients and samples. Subjects eligible for this study had histologically confirmed ovarian EMC or CCC with functioning stroma (Fig. 1). Patient and clinicopathological data, including age, menopause, International Federation of Obstetrics and Gynecology (FIGO) stage, histological subtype, histological grade, surgery (optimal, residual tumour $<1 \mathrm{~cm}$; suboptimal, residual tumour $\geq 1 \mathrm{~cm}$ ), serum oestrogen level, serum follicle-stimulating hormone (FSH) level, recurrence, and death, were reviewed. Serum levels of oestrogen (Eclusys E2 IV; Roche Diagnostics, Tokyo, Japan) and FSH (FSH II; Roche Diagnostics) were analysed by enzyme immunoassays. All patients had a follow-up period of at least three years. The study was approved by the Institutional Review Board of the Saitama Medical University International Medical Center (Saitama, Japan), and written informed consent was obtained from all patients.

Laser microdissection and DNA extraction. Formalin-fixed, paraffin-embedded sections $(10 \mu \mathrm{m})$ prepared from tumour tissue specimens were affixed to $2-\mu$ m-thick LCM Film glass slides (Membrane Slides PEN Membrane 2; Leica, Wetzlar, Germany) and stained with $0.05 \%$ toluidine blue solution (pH 2.5; Wako, Osaka, Japan). Stained sections were microdissected using a Leica LMD7000 laser microdissection microscope. Carcinoma cells and adjacent functional stromal cells were visualized under the microscope and were selectively detached by activation of the laser (Fig. 2). DNA was extracted using the Maxwell RSC DNA FFPE kit (Promega, Madison, WI, USA) according to the manufacturer's instructions.

Amplification and sequence analysis of ARIDIA, PIK3CA, KRAS, and PTEN. We analysed ARIDIA (exons 18 and 20), PIK3CA (exons 9 and 20), KRAS (exons 2 and 3), and PTEN (exons 5-8) sequences in DNA extracted from the whole tumours of 14 patients. For cases with mutations, carcinoma cells and functioning stromal cells were analysed separately to clarify the histological localization of the mutations. Target sequences were PCR-amplified using Accuprime Taq DNA Polymerase (Invitrogen, Carlsbad, CA, USA) on a 9800 Fast Thermal Cycler (Applied Biosystems, Foster City, CA, USA). Primer sequences are shown in Table I. The thermal cycles were as follows: $95^{\circ} \mathrm{C}$ for $10 \mathrm{~min}$, followed by 40 cycles of $94^{\circ} \mathrm{C}$ for $30 \mathrm{sec}, 60^{\circ} \mathrm{C}$ for $30 \mathrm{sec}$, and $68^{\circ} \mathrm{C}$ for $60 \mathrm{sec}$. Products were electrophoresed on a $2 \%$ agarose gel. Purified products were subjected to direct sequencing on an ABI PRISM 3100 (Applied Biosystems) using the ABI PRISM Big Dye Terminator Ver3.1 Cycle Sequencing kit according to the manufacturer's instructions. Sequencing was conducted twice to confirm reproducibility of the results.

\section{Results}

Patient characteristics. The median age of the 14 patients was 67 years (range, 52 to 85 years); 13 patients were postmenopausal, and one was in perimenopause. Serum oestrogen levels ranged from 10 to $129 \mathrm{ng} / \mathrm{ml}$, with a median of $51 \mathrm{ng} / \mathrm{ml}$, before surgery, but were reduced to less than $10 \mathrm{ng} / \mathrm{ml}$ in all available postoperative patients. Serum FSH levels ranged from 6 to $89 \mathrm{mIU} / \mathrm{ml}$, with a median of $32 \mathrm{mIU} / \mathrm{ml}$, preoperatively, but increased after surgery, ranging from 62 to $96 \mathrm{mIU} / \mathrm{ml}$ and with a median of $82 \mathrm{mIU} / \mathrm{ml}$. Ten patients had FIGO stage I cancer, two patients had stage II, and two patients had stage IV. Five patients with EMC had grade 1 cancer, and two patients with EMC had grade 2 cancer. Two patients with FIGO stage IV had suboptimal surgeries, and the others had optimal surgeries. Among CCC patients, four patients experienced recurrence, and three of them died of the disease. In EMC, there was no recurrence or death.

Localization of gene mutations. As shown in Table II, one patient had an ARIDIA mutation (7\%), two patients had PIK3CA mutations (14\%), three patients had KRAS mutations (21\%), and one patient had a PTEN mutation (7\%). In EMC, three patients had KRAS mutations (43\%), one patient had a PIK3CA mutation (14\%), and none of the patients had ARIDIA or PTEN mutations (0\%). In CCC, one patient had both PIK3CA and PTEN mutations (14\%), one patient had an ARIDIA mutation (14\%), and none of the patients had KRAS mutations $(0 \%)$.

In total, eight mutations were detected in whole tumours of five patients: ARIDIA (L2155L), PIK3CA (H1047R), KRAS (Q12V, E31K, Q61L), and PTEN (C105fs*8) (Table III). Seven of the eight mutations were detected in only carcinoma cells; thus, only one patient (case 4) had a $K R A S(\mathrm{E} 31 \mathrm{~K})$ mutation in both carcinoma and functioning stromal cells. The KRAS mutation in this case (Fig. 3) was repeatedly confirmed. In non-functioning stroma, composed of non-specific fibroblasts, no KRAS mutation was detected in this patient. Moreover, germline analysis of this patient revealed no KRAS mutation.

Trends in prognosis, hormone levels, and gene mutations. Five patients with high serum oestrogen levels $(\geq 50 \mathrm{ng} / \mathrm{ml})$ survived, whereas three patients (38\%) with low serum oestrogen levels $(<50 \mathrm{ng} / \mathrm{ml})$ died of the disease. In the four patients with recurrence, one patient (case 10) with a high serum oestrogen level $(88 \mathrm{ng} / \mathrm{ml})$ and low FSH level $(6 \mathrm{mIU} / \mathrm{ml})$ experienced disease-free survival after chemotherapy, but the other three patients died of the disease.

\section{Discussion}

In EOC, the cancer stroma is classified as either non-specific fibroblastic type or functioning stroma, the latter of which frequently occurs in postmenopausal EOCs. However, functioning stroma had not yet been thoroughly characterized in terms of histogenesis, response to chemotherapy, and prognosis. A close association between mucinous tumours and functioning stroma has been reported (19), and Katoh et al (20) found that functioning stroma is more common in EMCs resembling sex cord-stromal tumours. In the seven EMC and seven CCC 

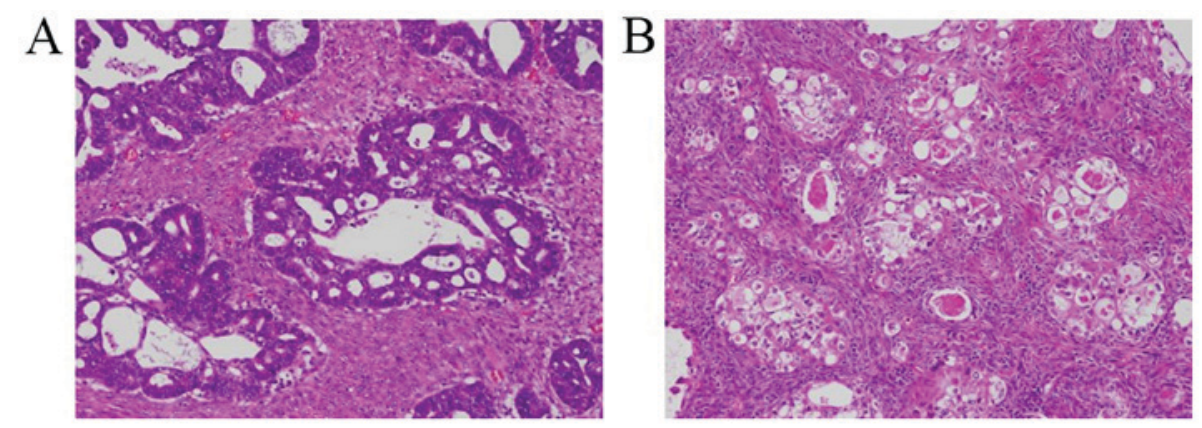

Figure 1. Histological findings. (A) Functioning stroma (case 4) in EMC and (B) CCC (case 10). Magnification, x20. EMC, endometrioid carcinoma; CCC, clear cell carcinoma.
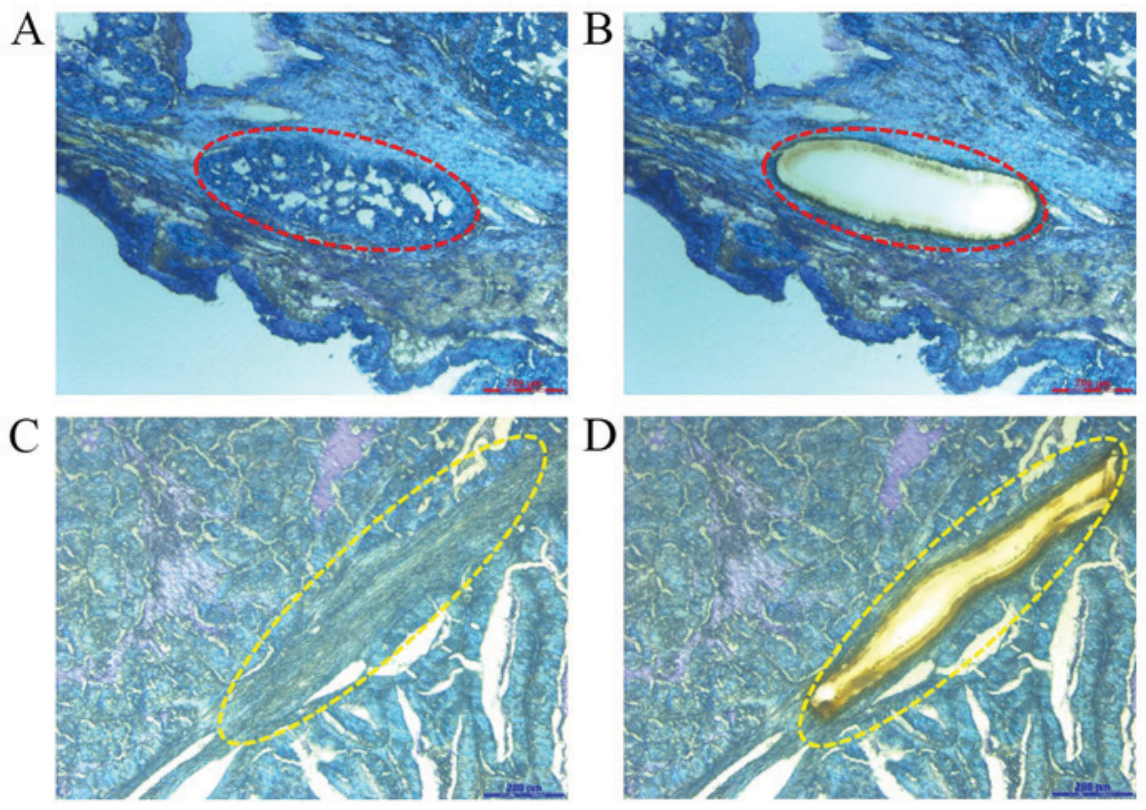

Figure 2. Laser microdissection from toluidine blue-stained sections (case 4). Carcinoma (A) prior to (red outlined area) and (B) following (red outlined area) dissection. Functioning stroma (C) prior to (yellow outlined area) and (D) following (yellow outlined area) dissection. Magnification, x20.
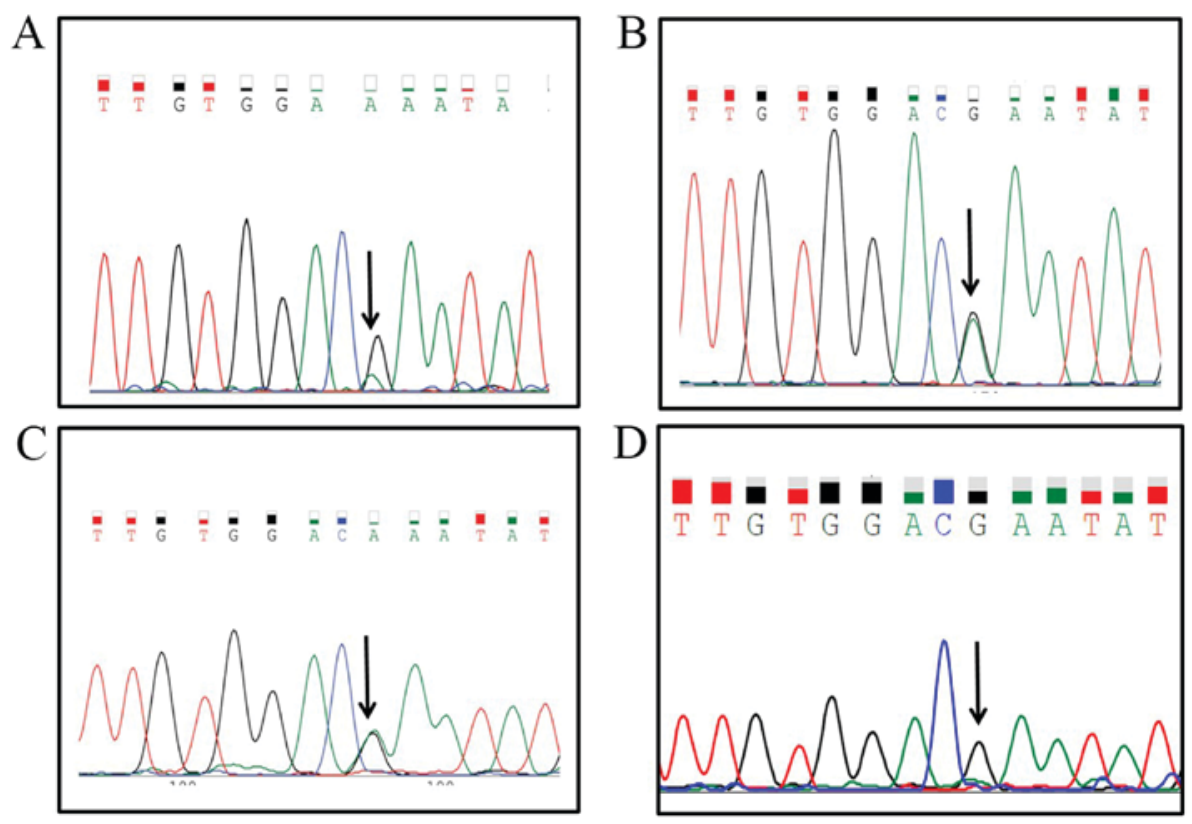

Figure 3. In case 4, the KRAS mutation was detected in (A) whole tumour, (B) carcinoma, and (C) functioning stroma, but not in (D) non-tumour tissues of the uterus. KRAS, Kirsten rat sarcoma viral proto-oncogene. 
Table I. Sequence information for primers used to amplify ARIDIA, PIK3CA, KRAS and PTEN.

\section{A, ARIDIA}

Gene $\quad$ Primer $\left(5^{\prime}-3^{\prime}\right)$

\section{Exon 18}

$\mathrm{S}$

AS

$\mathrm{S}$

AS

$\mathrm{S}$

AS

S

AS

$\mathrm{S}$

AS

$\mathrm{S}$

AS

Exon 20

$$
\mathrm{S}
$$

AS

$\mathrm{S}$

AS

S

AS

S

AS

S

AS

S

AS

S

AS

S

AS

S

AS

S

AS

TGGCATCTGTGGGCTTTATGT
CCATACTGGTTGTATACATCTTGCT
GGAGATGTACAGCGTGCCATA
TTGTGGTGGCATGTTTTGCTG
CAGAACCAATTTCCATTCCAGT
GTGTGCAGCATTTCATCTGTTC
GGGGCGTAATGACATGACCTAT
AATGTGATTCTGCATGCTTGGTG
CAAGGCCCCCTCCATCTAAC
TGCTAGGAGAGGTGCGGTTC
ATGCAGAATCACATTCCTCAGGTAT
GGCAGATTAGGCAACCGAATG

GGGGAGGTCTCTCAAGTCAAT

ATGGGAGCTGGACTAGACAC

GGACAGAGAACGCTACTGGAT

AATGGATCATTCTTCTGTACGATCT

GAGGAGAAGCTGATCAGTAAGTTTG

CTGCTGTTGTCACATGCTTCC

CTGAGCATATCCAGACCCACTTC

GCCTCTGAACTCTTAGCTCCATC

CAGCCACTATGGATGACATGTT

GGTGTTTGGACATCTCAAAGTCA

GCGTCTGTGTGTCCAATACCA

CACTCCACTTTGTTGCAGCTC

CAGGCACCACTAACTTATGAAAAGG

GAGTTTGCTGAGGGTTTCCAAGA

TCCTTTCCCCGCAGAGACT

CCAGGAGGTTGCCGATACTG

GTGCCATTGCAGTGCAGAA

GAGATGTCCAACAGCCGTGAT

TGGACGAGAACCACTCAGAGTTTAC AGGGCAACAGTCAGTTTCTAAGTTC

\section{B, PIK3CA}

\begin{tabular}{ll}
\hline Gene & \multicolumn{1}{c}{ Primer $\left(5^{\prime}-3^{\prime}\right)$} \\
\hline Exon 9 & \\
S & TGTAAAACGACGGCCAGTGGGAAA \\
& AATATGACAAAGAAAGC \\
AS & CAGGAAACAGCTATGACCTGAGAT \\
& CAGCCAAATTCAGTT
\end{tabular}

Exon 20

$\mathrm{S}$

AS
TGTAAAACGACGGCCAGTCTCAATG ATGCTTGGCTCTG

CAGGAAACAGCTATGACTGGAATCC AGAGTGAGCTTTC
Table I. Continued.

C, KRAS

\begin{tabular}{lc}
\hline Gene & \multicolumn{1}{c}{ Primer $\left(5^{\prime}-3^{\prime}\right)$} \\
\hline Exon 2 & \\
S & TAACCTTATGTGTGACATGTTC \\
AS & ATGCATATTAAAACAAGATTTACC \\
Exon 3 & \\
S & CTCCCTTCTCAGGATTCCTA \\
AS & AGTCCTCATGTACTGGTCCC \\
\hline
\end{tabular}

D, PTEN

Gene $\quad$ Primer $\left(5^{\prime}-3^{\prime}\right)$

Exon 5

S ACCTGTTAAGTTTGTATGCAAC

AS TCCAGGAAGAGGAAAGGAAA

Exon 6

S CATAGCAATTTAGTGAAATAACT

AS GATATGGTTAAGAAAACTGTTC

Exon 7

S TGACAGTTTGACAGTTAAAGG

AS GGATATTTCTCCCAATGAAAG

Exon 8

S ACACATCACATACATACAAGTC

AS GTGCAGATAATGACAAGGAATA

S, sense; AS, antisense; ARIDIA, AT-rich interaction domain 1A; PIK3CA, phosphatidylinositol-4,5-bisphosphate 3-kinase catalytic subunit $\alpha ;$ KRAS, Kirsten rat sarcoma viral proto-oncogene; PTEN, phosphatase and tensin homolog.

patients recruited in the present study, functioning stroma was identified histologically and endocrinologically. Five EOCs in four patients carried mutations in ARIDIA, PIK3CA, KRAS, and $P T E N$ in carcinoma cells, but not in functioning stromal cells. However, one case of ovarian EMC (case 4) harboured an identical KRAS mutation $(\mathrm{E} 31 \mathrm{~K})$ in both carcinoma and functioning stromal cells, whereas no mutation was detected in the tissue surrounding the tumour. Akahane et al (11) reported a case of ovarian EMC with carcinoma and stromal cells harbouring the same mutation in TP53 (R2489) as indicated by direct sequence analysis. Tuhkanen et al (12) reported 39 similar genetic alterations in carcinoma and stromal cells in 11 EOC tumours based on multiplex ligation-dependent probe amplification. These findings suggest that the cancer stroma may contain cells of epithelial origin that are generated by epithelial-mesenchymal transition and that this transformed tumour stroma may have an effect on epithelial-stromal cell interactions and tumorigenesis (12). Therefore, it may be possible to elucidate the mechanisms of tumour initiation by evaluating the association between carcinoma cells and functioning stromal cells. 


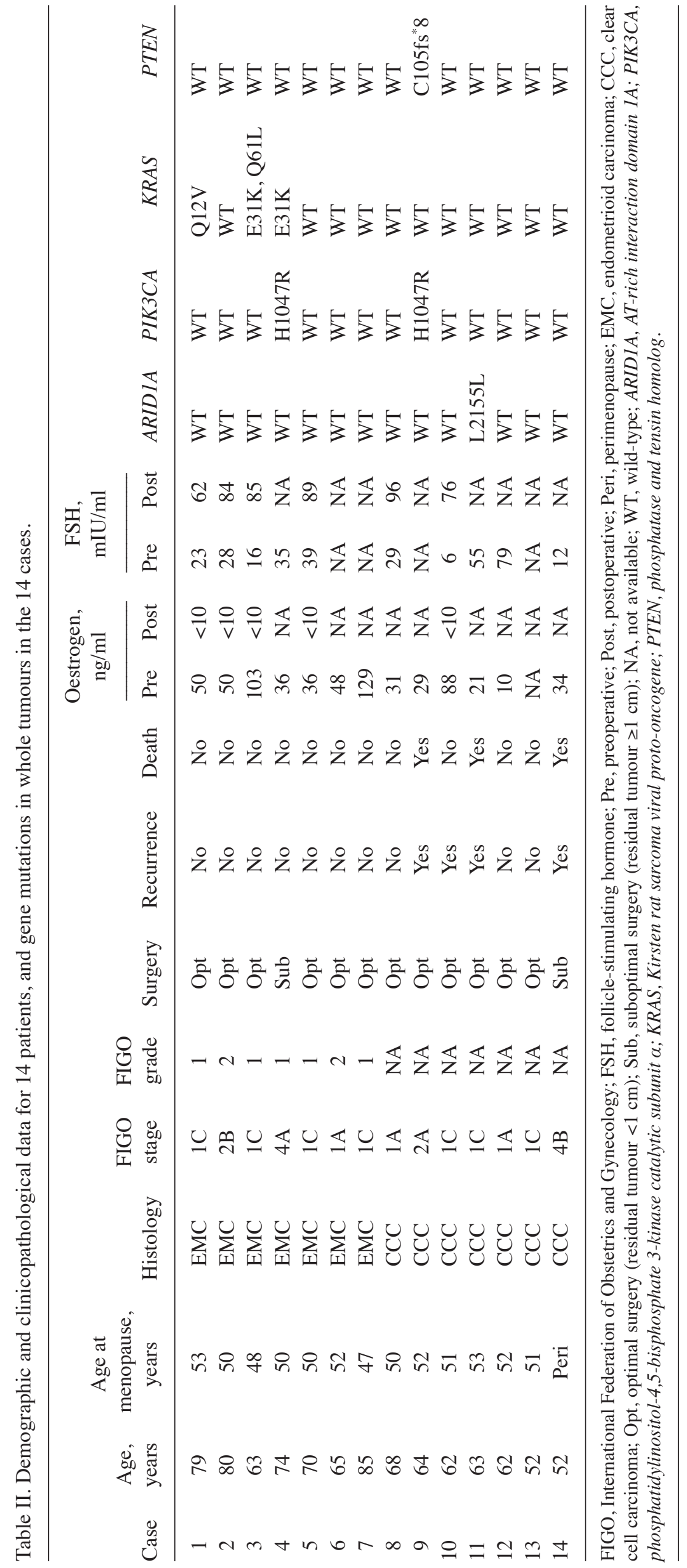


KRAS mutations are typically detected in $20-30 \%$ of EMC cases $(14,21)$; however, in the present study, EMC with functioning stroma had a higher frequency (43\%) of KRAS mutations. KRAS mutation is more common in endometriosis-associated EMC than in non-endometriosis-associated EMC (21). These findings suggest that EMC with functioning stroma may be associated with endometriosis. Mutations in ARIDIA, which are distributed evenly across the gene, are detected in $46 \%$ of ovarian CCC and $30 \%$ of EMC cases (16). In the present study, there was only one case of CCC (7\%) with ARIDIA mutation. However, we immunohistochemically confirmed the deletion of ARIDIA in eight of the 14 patients (57\%) (data not shown). This discrepancy may be explained by the fact that only exons 18 and 20 were sequenced in the present study. EOC patients with functioning stroma exhibited elevated serum oestrogen levels and reduced serum FSH levels. Following surgery, the levels of both hormones were restored to normal postmenopausal levels. Patients with high oestrogen levels $(\geq 50 \mathrm{ng} / \mathrm{ml}$ ) experienced no death from the disease, as demonstrated by one patient with EMC (case 10) who had a long period of disease-free survival after chemotherapy for recurrence; however, patients with low oestrogen levels ( $<50 \mathrm{ng} / \mathrm{ml}$ ) exhibited poor outcomes. Thus, the functioning stroma, characterized by morphological and endocrinological differentiation, may be related to the biological behaviours of cancer.

Our study had several limitations. The sample size was small and the range of genetic mutations analysed was narrow. In addition, the detection of identical mutations in both carcinoma and functioning stromal cells was not replicated, and differences between EMC with and without functioning stroma were not assessed. Therefore, this research should be considered a preliminary study. Confirmation of our findings in a larger population, including patients with and without functioning stroma, is warranted.

In conclusion, we are the first to report a case of EMC in which the same KRAS mutation was observed in both carcinoma cells and functioning stromal cells. This suggests that some regions of the tumour and stroma may have a common origin. Further studies are needed to clarify the molecular association of functioning stroma with carcinoma cells in a larger population.

\section{Acknowledgements}

Not applicable.

\section{Funding}

The present study was supported by Grants-in-Aid from the Ministry of Education, Science, Sports and Culture of Japan (grant nos. 15K08355 and 18K06997), a Sekiguchi Memorial Award (grant no. 18-C-1-02) and Saitama Medical University.

\section{Availability of data and materials}

All datasets generated in this study are available from the corresponding author upon reasonable request. 


\section{Authors' contributions}

MN took part in the study conception and design, as well as the acquisition, analysis and interpretation of data. MiY took part in the interpretation of data and drafting of the manuscript. KK and TK took part in the acquisition and analysis of data. MS and KI took part in the conception and design of the study, and revised the manuscript for important intellectual content. HK participated in the analysis of data and critically revised the manuscript for important intellectual content. MaY took part in the conception and design of the study, critically revised the manuscript for important intellectual content, and supervised the study.

\section{Ethics approval and consent to participate}

The study protocol was approved by the institutional review board of Saitama Medical University International Medical Center (Saitama, Japan), and written informed consent was obtained from all patients.

\section{Patient consent for publication}

The present study obtained written informed consent for publication from the all patients.

\section{Competing interests}

The authors declare that they have no competing interests.

\section{References}

1. Bhowmick NA and Moses HL: Tumor-stroma interactions. Curr Opin Genet Dev 15: 97-101, 2005.

2. Kim JB, Stein R and O'Hare MJ: Tumour-stromal interactions in breast cancer: The role of stroma in tumourigenesis. Tumour Biol 26: 173-185, 2005.

3. Tlsty TD and Coussens LM: Tumor stroma and regulation of cancer development. Annu Rev Pathol 1: 119-150, 2006.

4. Olumi AF, Grossfeld GD, Hayward SW, Carroll PR, Tlsty TD and Cunha GR: Carcinoma-associated fibroblasts direct tumor progression of initiated human prostatic epithelium. Cancer Res 59: 5002-5011, 1999.

5. Nagasaki T, Hara M, Nakanishi H, Takahashi H, Sato M and Takeyama H: Interleukin-6 released by colon cancer-associated fibroblasts is critical for tumour angiogenesis: Anti-interleukin-6 receptor antibody suppressed angiogenesis and inhibited tumour-stroma interaction. Br J Cancer 110: 469-478, 2014.

6. Liu J, Chen S, Wang W, Ning BF, Chen F, Shen W, Ding J, Chen W, Xie WF and Zhang X: Cancer-associated fibroblasts promote hepatocellular carcinoma metastasis through chemokine-activated hedgehog and TGF- $\beta$ pathways. Cancer Lett 379: 49-59, 2016.
7. Straussman R, Morikawa T, Shee K, Barzily-Rokni M, Qian ZR, Du J, Davis A, Mongare MM, Gould J, Frederick DT, et al: Tumour micro-environment elicits innate resistance to RAF inhibitors through HGF secretion. Nature 487: 500-504, 2012.

8. Kurose K, Gilley K, Matsumoto S, Watson PH, Zhou XP and Eng C: Frequent somatic mutations in PTEN and TP53 are mutually exclusive in the stroma of breast carcinomas. Nat Genet 32: 355-357, 2002.

9. Wernert N, Locherbach C, Wellmann A, Behrens P and Hügel A: Presence of genetic alterations in microdissected stroma of human colon and breast cancers. Anticancer Res 21: 2259-2264, 2001.

10. Paterson RF, Ulbright TM, MacLennan GT, Zhang S, Pan CX, Sweeney CJ, Moore CR, Foster RS, Koch MO, Eble JN and Cheng L: Molecular genetic alterations in the laser-capture-microdissected stroma adjacent to bladder carcinoma. Cancer 98: 1830-1836, 2003.

11. Akahane T, Hirasawa A, Tsuda H, Kataoka F, Nishimura S, Tanaka H, Tominaga E, Nomura H, Chiyoda T, Iguchi Y, et al: The origin of stroma surrounding epithelial ovarian cancer cells. Int J Gynecol Pathol 32: 26-30, 2013.

12. Tuhkanen H, Anttila M, Kosma VM, Heinonen S, Juhola M, Helisalmi S, Kataja V and Mannermaa A: Frequent gene dosage alterations in stromal cells of epithelial ovarian carcinomas. Int $\mathrm{J}$ Cancer 119: 1345-1353, 2006.

13. Permuth-Wey J and Sellers TA: Epidemiology of ovarian cancer. Methods Mol Biol 472: 413-437, 2009.

14. McConechy MK, Ding J, Senz J, Yang W, Melnyk N, Tone AA, Prentice LM, Wiegand KC, McAlpine JN, Shah SP, et al: Ovarian and endometrial endometrioid carcinomas have distinct CTNNB1 and PTEN mutation profiles. Mod Pathol 27: 128-134, 2014.

15. Murakami R, Matsumura N, Brown JB, Higasa K, Tsutsumi T, Kamada M, Abou-Taleb H, Hosoe Y, Kitamura S, Yamaguchi K, et al: Exome sequencing landscape analysis in ovarian clear cell carcinoma shed light on key chromosomal regions and mutation gene networks. Am J Pathol 187: 2246-2258, 2017.

16. Wiegand KC, Shah SP, Al-Agha OM, Zhao Y, Tse K, Zeng T, Senz J, McConechy MK, Anglesio MS, Kalloger SE, et al: ARID1A mutations in endometriosis-associated ovarian carcinomas. N Engl J Med 363: 1532-1543, 2010.

17. Kim SI, Lee JW, Lee M, Kim HS, Chung HH, Kim JW, Park NH Song YS and Seo JS: Genomic landscape of ovarian clear cell carcinoma via whole exome sequencing. Gynecol Oncol 148: 375-382, 2018.

18. Scully RE YR and Clement PB (eds): Tumors with functioning stroma. Tumors of the ovary, maldeveloped gonads, fallopian tube and broad ligament. In: Atlas of Tumor Pathology. Armed Forces Institute of Pathology, Washington, DC, pp373-378, 1998.

19. Kato N, Hayasaka T, Takeda J, Osakabe M and Kurachi H: Ovarian tumors with functioning stroma: A clinicopathologic study with special reference to serum estrogen level, stromal morphology and aromatase expression. Int J Gynecol Pathol 32: 556-561, 2013.

20. Katoh T, Yasuda M, Hasegawa K, Kozawa E, Maniwa J and Sasano H: Estrogen-producing endometrioid adenocarcinoma resembling sex cord-stromal tumor of the ovary: A review of four postmenopausal cases. Diagn Pathol 7: 164, 2012.

21. Stewart CJ, Leung Y, Walsh MD, Walters RJ, Young JP and Buchanan DD: KRAS mutations in ovarian low-grade endometrioid adenocarcinoma: Association with concurrent endometriosis. Hum Pathol 43: 1177-1183, 2012. 